\title{
IGCS Gynecologic Oncology Global Curriculum \& Mentorship Program in Mozambique: Challenges and Results of an Overseas Surgical Training Program
}

Dércia Changule ${ }^{1}$, Ricardina Rangeiro ${ }^{1}$, Siro Daud'1, Magda Ribeiro', Elvira Luis', Flora Mabota$^{1}$, Georgia Fontes Cintra², Renato Moretti-Marques ${ }^{3}$, Marcelo A. Viera ${ }^{4}$, Mila Pontremoli Salcedo ${ }^{5}, 6$, Ellen Baker ${ }^{6}$, Cesaltina Lorenzoni ${ }^{1,8,9}$, Kathleen M. Schmeler ${ }^{6}$, Andre Lopes ${ }^{7}$.

\begin{abstract}
Hospital Central de Maputo, Maputo, Mozambique1; Hospital Sirio Libanệs, Brasília, Brazil2; Hospital Israelista Albert Einstein, SP, Brazil|3; Hospital de Câncer de Barretos, Barretos, Brazil/4; Universidade Federal de Ciências da Saúde de Porto Alegre (UFCSPA)/Irmandade Santa Casa de Misericórdia, Porto Alegre, Brazil5; Departments of Gynecologic Oncology \& Reproductive Medicine from The University of Texas MD Anderson Cancer Center, Houston, Texas, USA Department of Gynecology, Instituto Brasileiro de Controle de Câncer, SP, Brasil7; Ministério da Saúde, Mozambique ${ }^{8}$, Faculdade de Medicina, Universidade Eduardo Mondlane ${ }^{9}$
\end{abstract}

\section{Objective}

To describe the implementation of the IGCS Gynecologic Oncology Global Curriculum \& Mentorship Program (Global Curriculum) in Mozambique.

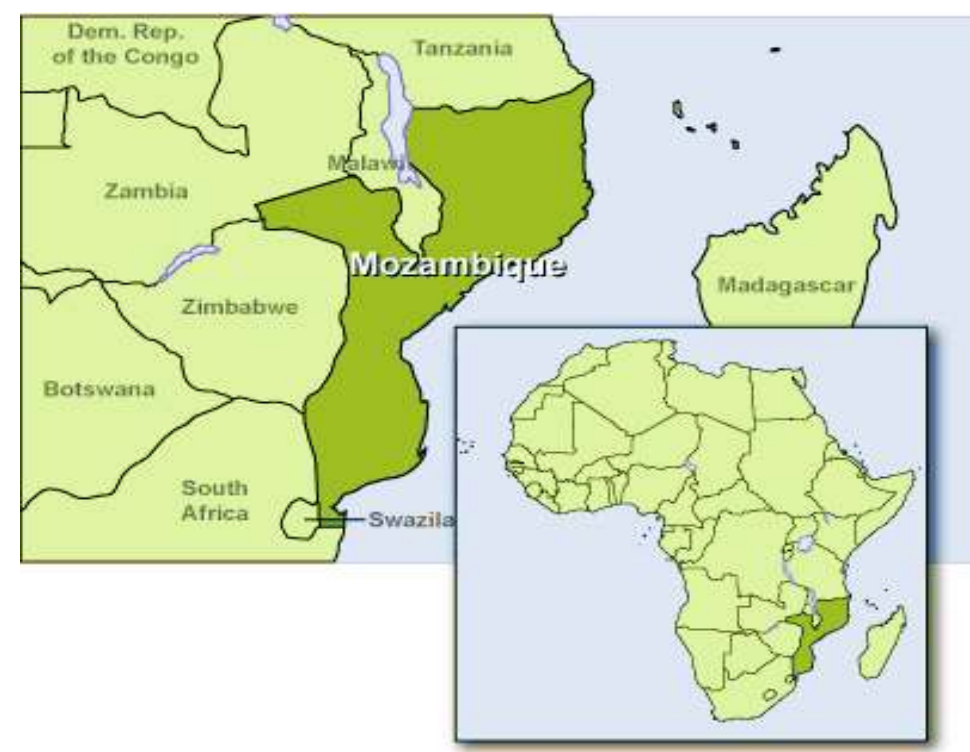

Source: https://www.mozambiqueembassy.ch
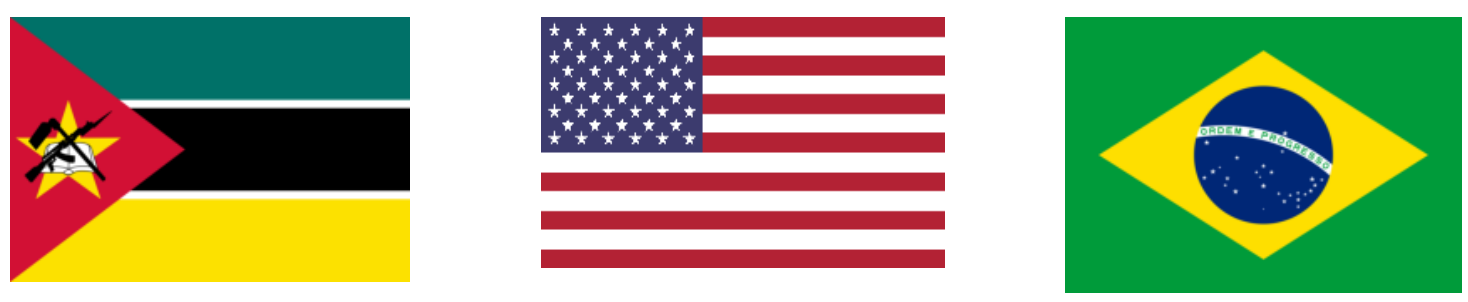

\section{Methods}

The Global Curriculum is a training program for regions of the world that do not have formal training in Gynecologic Oncology. The Mozambique program is a collaboration between Maputo Central Hospital, five Institutions in Brazil (Instituto Brasileiro de Controle do Cancer - IBCC, Santa Casa de Misericordia de Porto Alegre/UFCSPA, Hospital Albert Einstein, Hospital Sírio Libanês and Hospital do Câncer de Barretos) and the MD Anderson Cancer Center (USA).

In January 2016, three Obstetrician-Gynecologists were selected as the Global Curriculum fellows. They follow an on-line curriculum, receive quarterly visits from the International mentors, participate in monthly tumor boards using Project ECHO and enter case logs into the REDCap system.

\section{Results}

To date, there have been 10 visits to Mozambique. Each visit consists of didatic lectures, surgical training, multi-disciplinar care and the management of preinvasive disease. Between visits, monthly videoconferences are held to discuss patient cases.

A total of 101 surgeries have been perfomed, including 49 radical hysterectomies, 12 cold knife conizations and 14 radical vulvectomies. Seven colposcopies and LEEP courses were held with 274 attendees from all provinces of the country, 221 colposcopies and 35 LEEPs perfomed. In August 2018, a patient underwent radical hysterectomy and it was the first time this procedure was perfomed exclusively by Mozambicans surgeons.

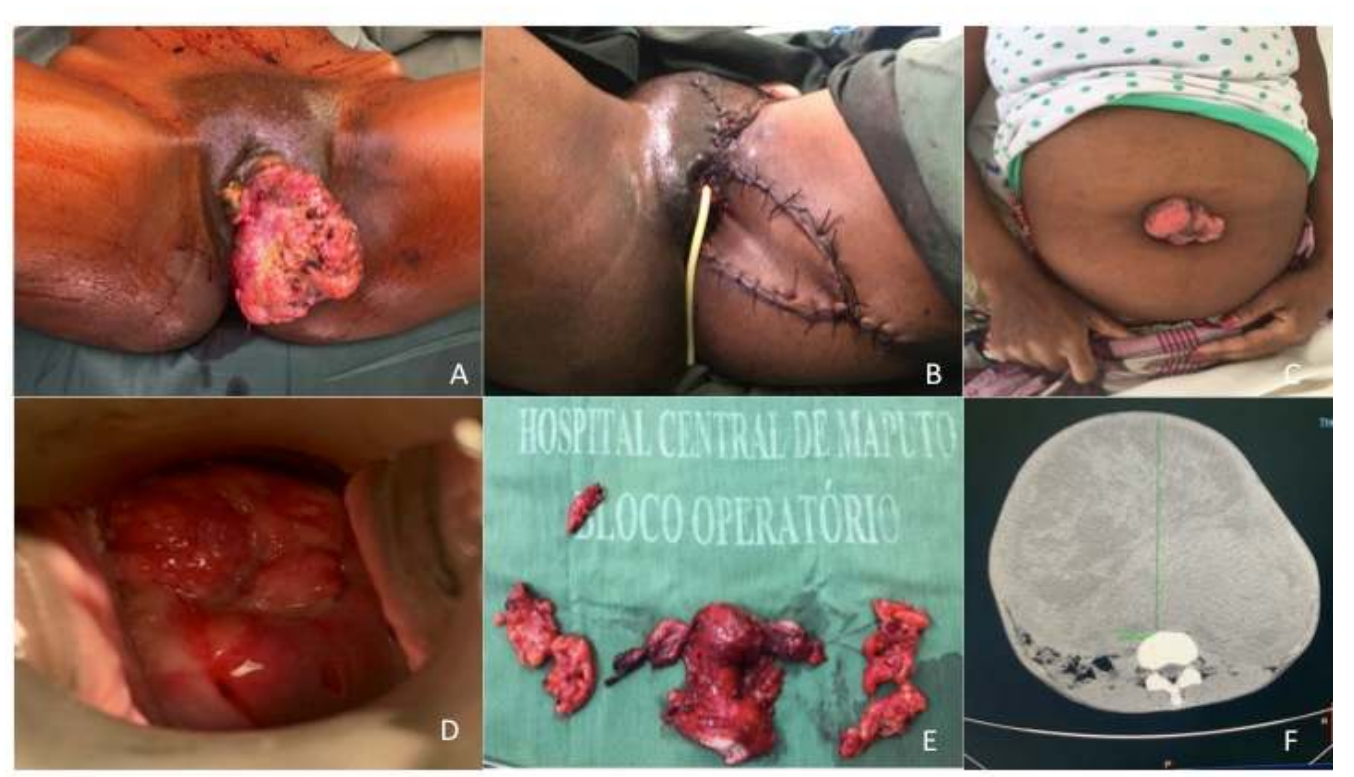

Figure - Phographs ilustrating surgical cases. A - HIV postive patient with vulvar cancer FIGO Stage IB. B- Final aspect after resection and vulvar flap reconstruction of patient in picture A. C - Patient presenting a "Syster Mary Joseph" nodule due to ovarian cancer. D - Locally advanced cervical cancer FIGO Stage IIA2, the patient was referred to neoadjuvant chemotherapy. E - Specimen from a radical Hysterectomy and pelvic lymphadenectomy for cervical cancer. F. Computed tomography showing a large uterine mass.

\section{Conclusions}

The IGCS model of surgical training is feasible and has already shown good results for the patients and fellows in Mozambique. 